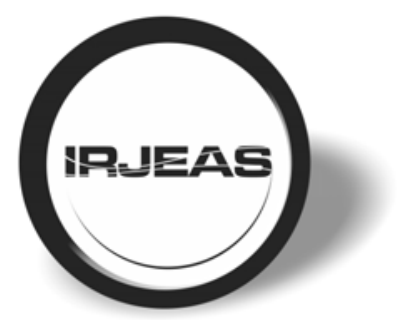

INTERNATIONAL RESEARCH JOURNAL

OF

ENGINEERING \& APPLIED SCIENCES

\title{
PSO WITH NEURAL NETWORK APPROACH FOR NETWORK SELECTION AND FAST DELIVERY HANDOVER ROUTE IN 5G
}

\author{
*B. Michael ${ }^{1}$, Diego Serrano² \\ 1,2 Department of Electronics \& Communication, Universitat Politècnica de Catalunya, Barcelona, Spain
}

\begin{abstract}
The enthusiasm for run resources has extended essentially with the presence of present day remote applications. Range sharing, considered as an essential instrument for 5G frameworks, is envisioned to address extend deficiency issue, achieve high data rate get to, and guaranteed Quality of Service (QoS). From the authorized system's point of view, the obstruction caused by all secondary users (SUs) ought to be limited. From optional systems perspective, there is a need to role out systems to SUs such that general impedance is diminished, empowering the settlement of a developing number of SUs. This paper presents a network and Fast Delivery Handover Route in order to less no of iteration to optimize fitness value and decision weight, in terms of RSSI, Loss, bandwidth, Speed, crossover rate and cost. To solve the optimization problem, Particle swarm optimization and Neural Network are used. At last, the paper is upheld by broad recreation comes about which show the adequacy of the proposed techniques in finding a close ideal arrangement.
\end{abstract}

Keywords- QoS, SU, PSO, Neural Network, 5G

\section{INTRODUCTION}

$\mathrm{T}$ The a decade ago has seen the sensational increment in the request of portable information because of the expansion in cell phones and adaptable applications. The interest for range assets has expanded significantly with the approach of current remote applications. Range sharing, considered as a basic component for $5 \mathrm{G}$ systems, is imagined to address range shortage issue, accomplish high information rate get to, and ensured nature ofbenefit. From the authorized system's point of view, the obstruction caused by every auxiliary client ought to be limited. From optional systems perspective, there is a need to allot systems to SUs such that general obstruction is decreased, empowering the convenience of a developing number of SUs.

The enhancement of design and weights of bolster for-ward Neural Systems is an intricate assignment of extraordinary significance in issues of regulated learning. In this work we break down the utilization of the Molecule Swarm Enhancement calculation for the streamlining of Neural System structures and weights pointing better speculation exhibitions through the making of a trade off between low compositional many-sided quality and low preparing blunders. For assessing these calculations we apply them to benchmark arrangement issues of the medicinal field. The outcomes demonstrated that a PSO-PSO based approach speaks to a substantial other option to improve weights and models of MLP Neural Systems.

The utilization of assets in remote sensor systems is generally very identified with the execution of assignment which devours sensible working and correspondence data transmission. To dispense the workload of each undertaking to appropriate hubs in a productive way an errand designation is required. Be that as it may, it is a run of the mill issue in the region of elite processing. Yet, this can be overcome, by applying the power and asset designation .In this paper the power and asset distribution utilizing altered double molecule swarm enhancement in neural systems should be possible so as to get the streamlined system. In this the power and asset distribution assumes an imperative part to beat the blurring channel and the cochannel obstruction. By utilizing the altered twofold molecule swarm streamlining the merging rate is to a great extent enhanced with the exchange capacity and position refreshing recipe and furthermore the assorted variety of particles is enhanced and the issue of neighbourhood minima is maintained a strategic distance from with the change activity.

In this work we apply the Molecule Swarm Streamlining calculation to the worldwide enhancement with $\mathrm{NN}$ and association weights. In this approach, PSO Neural Network for Network Selection and Fast Delivery Handover Route the two processes of connection weights optimization are interleaved for a number of iterations, to optimize fitness value and decision weight, in terms of RSSI, Loss, bandwidth, Speed, crossover rate and cost.

\section{BACKGROUND}

\section{A. Neural Networks}

These neural networks has an information layer, covered up or a yield layer, in which these layers are the interconnections among the neurons and every neuron included numerous data sources,

Weights and a solitary yield and further each layer have an exchange work which is in charge of mapping its contribution to yield. Normally this is a versatile framework in which it gets an arrangement of information sources, forms the information and gives the yield. These neural systems are to be prepared before utilizing 
them. This preparation procedure is finished by giving the right responses for a given arrangement of information sources and by modifying the weights in light of the reaction given by the network.

\section{B. Binary Particle Swarm Optimization (BPSO)}

In BPSO a potential answer for the issue is spoken to by a molecule. It has a twofold position vector and a speed vector and in which every molecule keeps up a record of its own best position and all in all of the swarm particles it keeps up a worldwide best position. Amid the emphasess every molecule can be refreshed by thinking about its own best position and a separation to the worldwide best position. It is refreshed by methods for exchanging between the 0 and 1 speed esteems.

In [11], in which, design, weights and other MLP parameters are enhanced by hereditary calculations. In [4], different methods in light of GA used to enhance MLPs are analyzed for design grouping issues. What's more, in [2], Zhang and Shao present a procedure completely in view of the standard PSO for engineering and weight improvement with few insights about execution and results got. Our work is roused by Zhang and Shao's approach however presents the weight rot heuristic [9] in the weight alteration process trying to get more speculation control. For assessing the proposed calculation we utilized benchmark characterization issues of the medicinal field (Tumor, Diabetes and Heart) acquired from the store Problem1 [8].

\section{PROBLEM IDENTIFICATION}

- More no of iteration make system complex and it take more time to find different value.

- Population size is less.

\section{PROPOSED SYSTEM}

- The aim is to achieve less no of iteration to optimize fitness value and decision weight, in terms of RSSI, Loss, bandwidth, Speed, crossover rate and cost.

- $\quad$ Particle Swarm Optimization and Neural Network are used to solve the optimization problem and Network Selection and Fast Delivery Handover Route.

\section{A. Particle Swarm Optimization}

The PSO advancement procedure is a stochastic hunt through a ndimensional issue space pointing the minimization (or amplification) of the target capacity of the issue. The PSO was made by Kennedy and Eberhart through the endeavor to graphically recreate the social conduct of a rush of winged creatures traveling to assets.

In PSO, a swarm of particles is kept, where every single one of them speaks to a point in the arrangement space. The particles of the swarm travel through that space contemplating the best position went by the individual and the best position went by the whole populace.
Give $\mathrm{s}$ a chance to be the swarm measure, $\mathrm{n}$ be the measurement of the advancement issue and $t$ the present moment, every molecule 1 $\leq \mathrm{i} \leq \mathrm{s}$ has a position $\mathrm{xi}(\mathrm{t}) \in \mathrm{Rn}$ in the arrangement space and a speed vi(t) $\in R n$ which controls the bearing and size of its development. Likewise, every molecule keeps in memory the best individual position $y i(t) \in R n$ went to un-til the moment $t$, and the entire swarm keeps in memory the best position $\mathrm{y}^{\wedge}(\mathrm{t}) \in \mathrm{Rn}$ went by every one of the particles. The conditions eq. (1) and eq. (2) portray, separately, how the new speed and the new position of a molecule are hinder mined.

$$
\begin{aligned}
& \text { vij }(t+1)=w v i j(t)+c 1 r 1(y i j(t)-x i j(t))+ \\
& +\quad c 2 r 2(y j(t)-x i j(t)) \\
& x i j(t+1)=x i j(t)+v i j(t+1)
\end{aligned}
$$

The scalar $\mathrm{w}$ is the idleness weight (force term) which duplicates the earlier speed of the molecule (moment $t$ ) and controls the level of investigation of the pursuit. The qualities $\mathrm{r} 1$ and $\mathrm{r} 2$ are uniform irregular factors taken from $\operatorname{Uij} 1(0,1)$ and $\operatorname{Uij} 2(0,1)$, separately. The individual and worldwide speeding up coefficients, $0<\mathrm{c} 1$, $\mathrm{c} 2 \leq 2$, separately, have settled and measure up to esteems, and are in charge of taking control of how far a molecule can move in a solitary cycle. The standard PSO calculation is exhibited in [7]. Fast merging in unimodal capacities, with great achievement rate, and untimely meeting in multimodal capacities are properties often ascribed to the standard PSO calculation [6].

\section{B. The PSO-PSO Approach}

The approach used to enhance weights and structures of MLP neural systems depends on the interleaved execution of two PSO calculations, one for weight streamlining (inward PSO) and the other for engineering advancement (outer PSO).

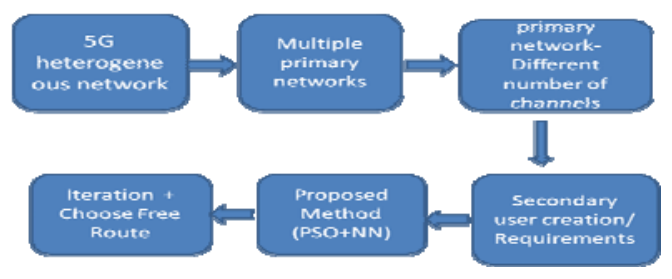

Fig.4.1 Block Diagram of Process

In our proposed system, we consider a $5 \mathrm{G}$ heterogeneous system that consolidates numerous essential systems. At any given moment, every essential system has an alternate number of channels accessible for the SUs, and each channel has an alternate limit. Each system has a few imperatives as far as obstruction, membership expenses, and limit. SUs determine their necessities as far as the base information rate and the most extreme membership charge that they will pay. 
Based on SU requirements and primary network constraints, we employ the PSO and the NN to find a near-optimal solution. The goal of this paper is to find a solution such that the overall less no of iteration to optimize fitness value and decision weight, in terms of RSSI, Loss, bandwidth, Speed, crossover rate and cost. Particle Swarm Optimization and Neural Network are used to solve the optimization problem and Network Selection and Fast Delivery Handover Route.

\section{ALGORITHM}

PSO NN algorithm for simultaneous optimization of weights Error and Fast Delivery Handover Route

Section 0: Make a Loop - Create a matrix for PSO to use 'RSSI', 'Loss', 'BW', 'Speed', 'RTT',' Cost' These are the six equivalent RF environments for each deficit above

Section I: Make a Name -Initialization matrix index

Section II: Make a Connection -connection matrix, nothing connected Section III: Make a Route - Prune the connection matrix with limited multiple connection $\mathrm{M}$

Section IV: Make a Bang-Now the random change of route or schedule happen!

Section V: Make a Rescue-Now trying to hand over the system, using hope matrix

Section VI: Make an Exit-Now trying to go with new network, using exit matrix

Section VII: Make a Final -RDBMS server fails rate Ms Section VIII: NN Train -run many times, to train neural network

\section{RESULT AND SIMULATION}

For simulating the results the software that is being used is the MATLAB R2014a.The following are the simulation results for Network Selection and Fast Delivery Handover Route in the PSO neural networks.

The Neural Network iteration method is capable of calculating the maximum of the thousand's of the nodes. In this each node depends on the previous node for the optimal power allocation. For example consider a single node and let us assume 10secs as duration for each node and if we consider for the 10 nodes then the time should be of 100 secs. So in this it calculates for each and every node and assumes the value itself when there is the multiple numbers of the nodes.

In the random instances method the calculation will be done completely in the random manner and finally from the above graph the optimal power allocation is obtained.

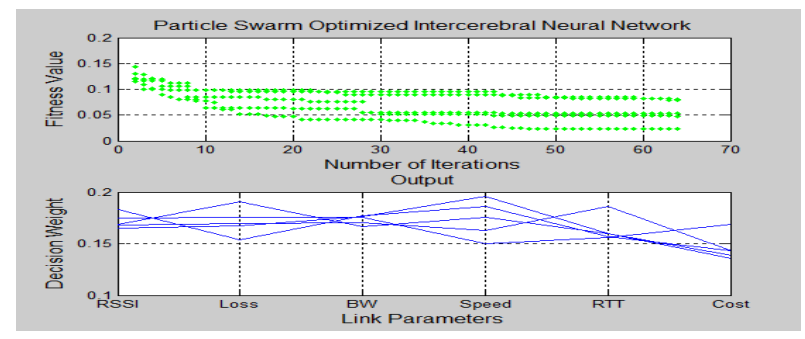

(a)

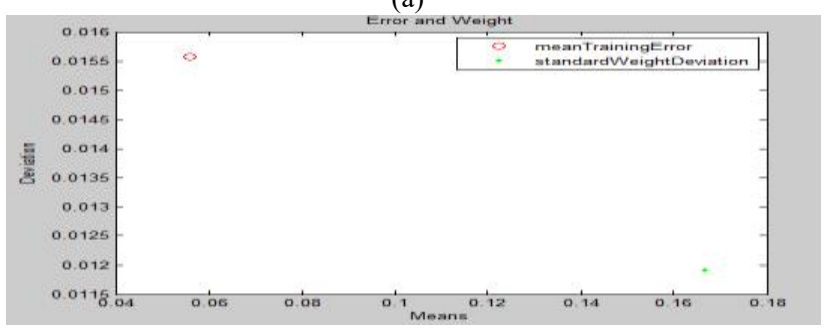

(b)

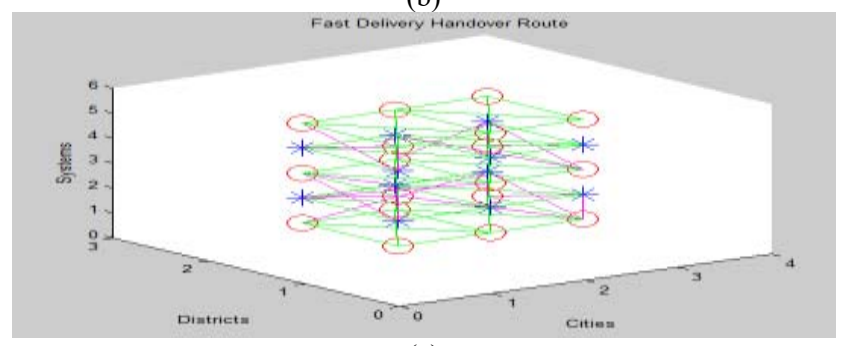

(c)

Fig 6.1 (a) PSO -NN for Iteration and Error (b) Training Error and SWD (c) Fast Delivery HR

Table 6.1. Parameters value

\begin{tabular}{|l|l|}
\hline PARAMETERS & PROPOSED WORK \\
\hline Method & Hybrid(PSO+NN) \\
\hline Iteration & $65-70$ \\
\hline Population size & PSO- 30 \& NN- 30 \\
\hline Inertial Weight & $1-6$ \\
\hline Cross over Rate & 0.4 \\
\hline
\end{tabular}

From the table- 6.1 it can be said that as the number of the iteration is decreasing as well as population size is increasing, inertial weight balance is increasing and cross over rate is decreasing.

Table 6.2. Simulation Parameters

\begin{tabular}{|l|c|}
\hline Mean Epoch & 34.3529 \\
\hline Std Epoch & 19.5378 \\
\hline Mean Err & 0.0558 \\
\hline
\end{tabular}


International Research Journal of Engineering \& Applied Sciences, IRJEAS

www.irjeas.org, ISSN(O): 2322-0821, Volume 9 Issue 3, Jul 2021-Sep 2021, Page 01-04

\begin{tabular}{|l|c|} 
& \\
\hline Std Err & 0.0156 \\
\hline Mean Wei & 0.1667 \\
\hline Std Wei & 0.0119 \\
\hline
\end{tabular}

\section{CONCLUSION}

In this work the Network Selection and Fast Delivery Handover Route in the PSO neural networks is proposed. By using these approaches in the neural network the convergence speed is increased by the position of updating the previous node and the network is optimized and it also shows the better energy efficiency. The simulation results also show the better performance in less no of iteration to optimize fitness value and decision weight, in terms of RSSI, Loss, bandwidth, Speed, crossover rate and cost to Network Selection and Fast Delivery Handover Route. As future works, we consider the fusion of the two interleaved tasks (inner PSO and outer PSO) in a single PSO searching for weights and architectures as a truly simultaneous optimization process. Another interesting possibility is the addition of a connectivity pattern optimization process to the PSO-PSO algorithm, since this approach has presented good generalization performance in other works.

\section{REFERENCES}

[1]. Najam ul Hasan, Waleed Ejaz, Naveed Ejaz, Hyung Seok Kim, Alagan Anpalagan, and Minho Jo, "System Choice and Channel Allotment for Range Partaking in 5G Heterogeneous Systems", IEEE Access 2016

[2]. S. Chen and J. Zhao, "The prerequisites, difficulties, and innovations for $5 \mathrm{G}$ of earthbound portable media transmission," IEEE Commun. Mag., vol. 52, no. 5, pp. 36_43, May 2014.

[3]. M. Iwamura, "NGMN see on 5G design," in Proc. IEEE 81st Veh. Technol. Conf. (VTC Spring), Glasgow, Scotland, May 2015, pp. 15 .

[4]. H. Droste et al., "The METIS 5G architecture:Asummary of METIS deal with 5G structures," in Proc. IEEE 81st Veh. Technol. Conf. (VTC Spring), Glasgow, Scotland, May 2015, pp. 1_5.

[5]. P. K. Agyapong, M. Iwamura, D. Staehle, W. Kiess, and A. Benjebbour, "Design contemplations for a 5G arrange engineering," IEEE Commun. Mag., vol. 52, no. 11, pp. 65_75, Nov. 2014.

[6]. A. M. Akhtar, X.Wang, and L. Hanzo, "Synergistic range partaking in 5G HetNets: An orchestrated SDN-empowered approach," IEEE Commun. Mag., vol. 54, no. 1, pp. 40_47, Jan. 2016.

[7]. N. Bhushan et al., "Network densication: The predominant topic for remote development into 5G," IEEE Commun. Mag., vol. 52, no. 2, pp. 82 89, Feb. 2014.

[8]. W. Ejaz and M. Ibnkahla, "Machine-to-machine interchanges in subjective cell frameworks," in Proc. IEEE Int. Conf. Universal Remote Broadband (ICUWB), Montreal, QC, Canada, Oct. 2015, pp. 1 5.

[9]. C. Rattaro, L. Aspirot, and P. Belzarena, "Analysis and portrayal of dynamic range partaking in subjective radio systems," in Proc. IEEE Int. Remote Commun. Versatile Comput. Conf. (IWCMC), Dubrovnik, Croatia, Aug. 2015, pp. 166171.
[10]. W. Ejaz, N. ul Hasan, S. Lee, and H. S. Kim, “I3S: Smart range detecting plan for intellectual radio systems," EURASIP J. Remote Commun. Netw., vol. 2013, no. 1, pp. 1_10, Dec. 2013.

[11]. W. Ejaz, N. Ul Hasan, and H. S. Kim, "Distributed helpful range detecting in intellectual radio for impromptu systems," Comput. Commun., vol. 36, no. 12, pp. 1341 1349, Jul. 2013.

[12]. M. Ju and K. Kang, "Cognitive radio systems with optional system determination," IEEE Trans. Veh. Technol., vol. 65 , no. 2 , pp. 966 972, Feb. 2016.

[13]. S. Andreev et al., "Intelligent get to organize determination in merged multiradio heterogeneous systems," IEEE Remote Commun., vol. 21, no. 6, pp. 86_96, Dec. 2014. 